\title{
Local surface pressure gradients observed during the transfer of mixed behenic acid/pentadecanoic acid Langmuir films
}

\author{
Stéphane Alexandre, Céline Lafontaine and Jean-Marc Valleton* \\ UMR 6522 Université de Rouen - CNRS, Université de Rouen, 76821 Mont-Saint-Aignan, France
}

\begin{abstract}
A mixed Langmuir film of behenic acid and pentadecanoic acid has been elaborated on water. This film contained domains and appeared very rigid and stable, but a vertical transfer onto a solid substrate was difficult because of this rigidity. A horizontal transfer technique was needed for efficiently transferring this film. The existence of pressure gradients in this film is discussed.
\end{abstract}

Keywords: fatty acids, Langmuir films, pressure gradients

\section{INTRODUCTION}

Fatty acids are good candidates for modelling biological membranes and the interactions occurring between lipids and proteins [1-2]. In particular, it is possible to form Langmuir films at the air/water interface when the hydrophobic chains are long enough to ensure a high water insolubility of the fatty acids. When transferred on a solid substrate by the Langmuir-Blodgett technique [3], the films can be studied by different spectroscopies, and by scanning force microscopy [1]. A problem of interest is to study the formation of lipid domains which may play a key rôle in biological processes [4]. It is possible to model this behaviour in Langmuir monolayers, by using at least two lipids [5-8], for example two fatty acids differing only by their chain length [9].

In this paper we report an initial study of Langmuir films constituted of two fatty acids, behenic acid and pentadecanoic acid. These first investigations led us to obtain original results, and in particular to observe a great rigidity of the mixed film. Scanning force microscopy confirmed the existence of microscopic domains stable for long times in these mixed films transferred on muscovite. The existence of pressure gradients in the film is discussed.

\section{MATERIALS AND METHODS}

A laboratory-built Langmuir-Blodgett trough made of PTFE (dimensions $50 \times 10.9 \mathrm{~cm}$; volume of the liquid phase $550 \mathrm{~cm}$ ) was used for the preparation of the samples. It was equipped with two plunging mobile barriers to ensure symmetrical compression of the amphiphilic molecules. A Wilhelmy balance was used for measuring the interfacial pressure (precision $\pm 0.1 \mathrm{mN} / \mathrm{m}$ ). The trough was enclosed in a cabinet in which the temperature was maintained at $22{ }^{\circ} \mathrm{C}$.

The elaboration of mixed films of behenic acid (BA) and pentadecanoic acid (PDA) was achieved using a pure

\footnotetext{
* Author for correspondence.
}

water subphase. The water was obtained by a Millipore system involving deionization, reverse osmosis and filtration (Milli-RO + Milli-Q).

BA and PDA obtained from Sigma, (purity 99\%) were prepared as $10^{-3} \mathrm{M}$ solutions in chloroform, and the BA / PDA mixture was prepared as an equimolar $10^{-3} \mathrm{M}$ solution; in each case, $0.1 \mathrm{ml}$ of the solution was spread with a capillary micropipette (Nichiryo) in order to study the pressure/area isotherms.

After spreading the solutions, 15 min were allowed for solvent evaporation and for relaxation of the system. Then the film was compressed (rate of compression was $0.5 \mathrm{~cm} / \mathrm{min}$ ) to obtain pressure/area isotherms. For the mixture, $24 \mathrm{~h}$ were allowed for complete stabilization of the domains in the films compressed to $15 \mathrm{mN} / \mathrm{m}$.

The transfer was performed according to the Langmuir-Schaefer technique (horizontal method) at $15 \mathrm{mN} / \mathrm{m}$, this pressure being maintained by a feedback loop. The transfers were performed on $9 \times 9 \mathrm{~mm}$ square muscovite mica pieces, $200 \mu \mathrm{m}$ thick, obtained from Métafix (Montdidier, France). The muscovite samples were freshly cleaved before any transfer, and used without any particular processing. They were dipped into the subphase before fatty acid solution spreading and covered by one monolayer during the upward translation (rate of vertical transfer was $1 \mathrm{~cm} / \mathrm{min}$ ). The transfer ratio was estimated to be $0.9 \pm 0.1$.

The scanning force microscopy image presented in this paper in the contact mode was obtained with a Nanoscope II from Digital Instruments, with a $180 \mathrm{~mm}$ piezoelectric scanner. The cantilever used was characterized by a low spring constant $(0.06 \mathrm{~N} / \mathrm{m})$. A standard tip of silicon nitride was used. The measurement was performed with the feedback loop on (constant force: $10^{-9}$ to $10^{-8} \mathrm{~N}$ ). The scan rate was $2.5 \mathrm{~Hz}$ (scan lines per second). All investigations were performed in air.

\section{RESULTS}

Pressure-area isotherms of BA, PDA and BA/PDA are presented in fig. 1. The BA isotherm is characterized by two liquid-condensed (LC) phases (transition at $21 \mathrm{mN} / \mathrm{m}$ ) followed by a solid (S) phase (transition at 


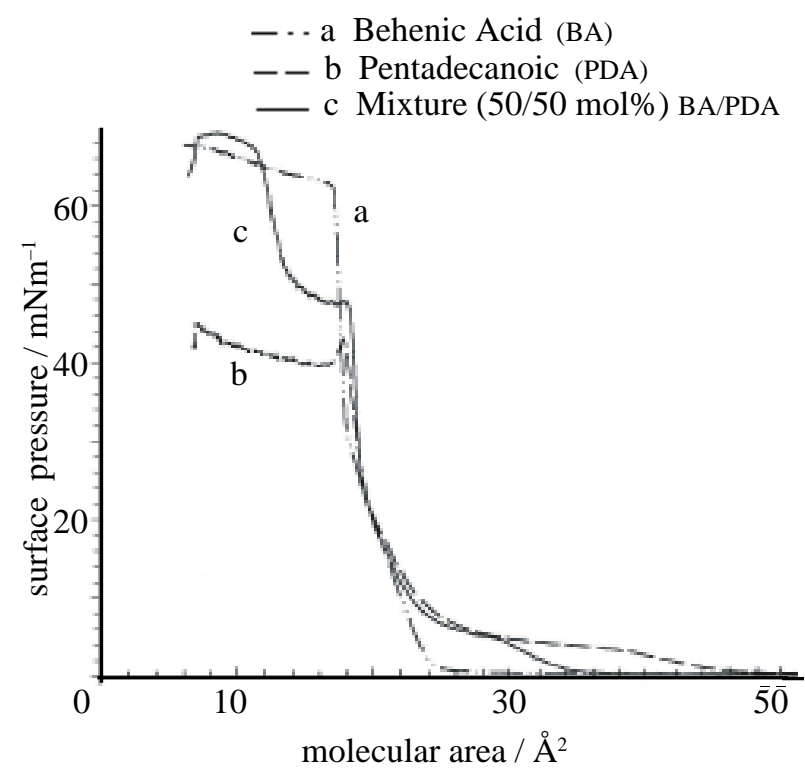

Figure 1. Pressure/area isotherms of Langmuir films: a) behenic acid, b) pentadecanoic acid, c) equimolar mixture of behenic and pentadecanoic acids.

$31 \mathrm{mN} / \mathrm{m})$. The PDA isotherm is characterized by a long phase transition $(4-5 \mathrm{mN} / \mathrm{m})$ between a liquid-expanded (LE) and a liquid-condensed phase. The BA/PDA mixture is characterized by an isotherm lying between the BA and PDA isotherms. On this isotherm it is possible to observe characteristics of both fatty acids: the LE/LC transition of PDA, the LC/S transition of BA, and the PDA and BA collapse pressures. These observations are consistent with strong immiscibility of the two fatty acids. The mixed BA/PDA Langmuir films revealed themselves to be very stable.

Because of their great rigidity, these films could not be transferred properly onto muscovite by the classical Langmuir-Blodgett technique: when the muscovite sample was translated vertically through the interface, the surface pressure remained constant, but the mobile barriers did not move to compensate for the removal of the film transferred on the sample. An investigation of this sample by scanning force microscopy showed that film transfer indeed occurred, although its quality was exceedingly poor.

It was however possible to transfer these PDA/BA films onto muscovite by the Langmuir-Schaefer technique. During the horizontal transfer, the same behaviour of the mixed film was observed: when the muscovite sample was lifted horizontally through the interface, the surface pressure remained constant and the mobile barriers did not move to compensate for the removal of the film. The BA/PDA Langmuir films transferred on muscovite, studied by atomic force microscopy, exhibit large domains (fig. 2) as expected from the isotherms.

At the end of every experiment with the mixed film, when the film was removed by aspiration prior to cleaning the air/water interface, we observed that the surface pressure began to decrease very late during the procedure, when a great part of the film was already removed.

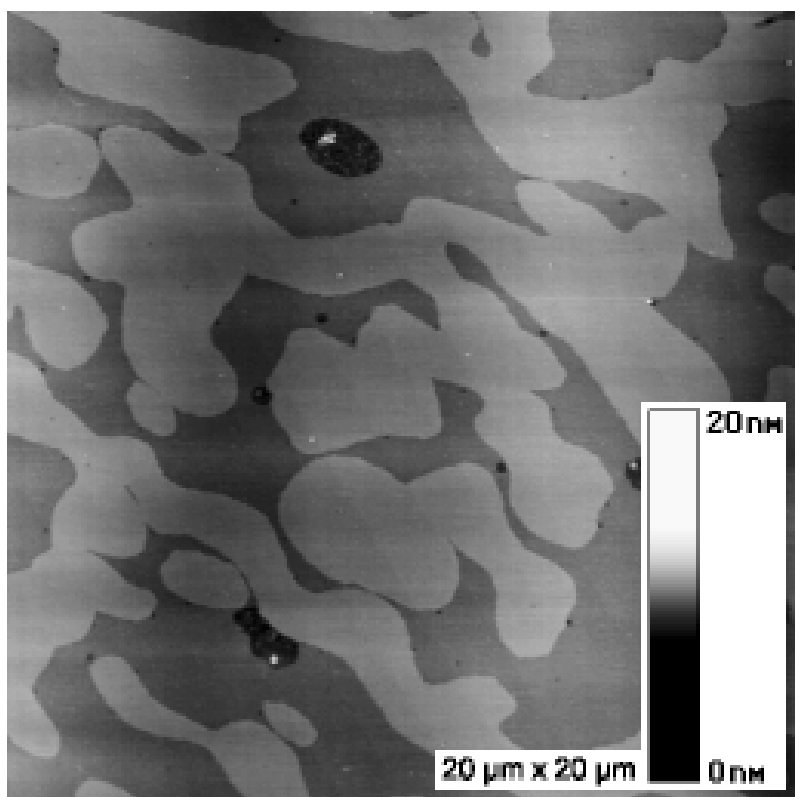

Figure 2. Scanning force microscopy (contact mode) image of a mixed behenic acid/pentadecanoic acid film transferred horizontally onto muscovite mica at a surface proessure of $15 \mathrm{mN} / \mathrm{m}$. Image size is $20 \times 20 \mathrm{~mm}$. The image is presented in height mode. Dark colours represent low zones, light colours high zones.

These observations clearly demonstrate the existence of zones with very different surface pressures: quite likely less than $1 \mathrm{mN} / \mathrm{m}$ in the zones in which the mixed film had been removed by transfer or aspirated for cleaning the interface, and $15 \mathrm{mN} / \mathrm{m}$ for the other zones.

\section{DISCUSSION}

Surface pressure gradients have already been observed in insoluble macromolecular films $[10,11]$ and in monoparticulate layers [12] at air/water interfaces. They have been interpreted as due to the cohesiveness of the layers.

In our case, the origin of the pressure gradient is probably similar: the rigidity of the film is such that the removal of matter due to the transfer which should result in a pressure decrease is not sensed by the pressure transducer if it is placed far from the zone of transfer. Hence, different local pressures might well coexist in the mixed film. Already at the end of compression to high surface pressures (presumably above some threshold value) there is probably a pressure gradient along the trough between the zone of compression and the more distant zones. It remains an open question whether PDA and BA are really totally immiscible, or whether partial miscibility, even if only slight, plays a rôle in the phenomenon. 


\section{REFERENCES}

1. Alexandre, S., Dubreuil, N., Fiol, C., Lair, D., Sommer, F., Tran-Minh Duc, Valleton, J.-M. Thin Solid Films 293 (1997) 295.

2. Fujiwara, I., Ohnishi, M., Seto, J. Langmuir 8 (1992) 2219.

3. Blodgett, K.B., J. Am. Chem. Soc. 157 (1935) 1007

4. Norris, V. Mol. Microbiol. 16 (1995) 1051.

5. Luzardo, M. del C., Peltzer, G., Disalvo, E. A. Langmuir 14 (1998) 5858.

6. DeWolf, C., Leporatti, S., Kirsch, C., Klinger, R., Brezesinski, G. Chem. Phys. Lipids 97 (1999) 129.
7. Garidel, P., Blume, A. Biochim. Biophys. Acta 1371 (1998) 83.

8. Koynova, R., Tenchov, B., Rapp, G. Chem. Phys. Lipids 88 (1997) 45.

9. Ekelund, K., Sparr, E., Engblom, J., Wennerström, H., Engström, S. Langmuir 15 (1999) 6946.

10. Peng, J.B., Barnes, G.T. Langmuir 6 (1990) 578.

11. Peng, J.B., Barnes, G.T., Abraham, B.M. Langmuir 9 (1993) 3574.

12. Máté, M., Fendler, J.H., Ramsden, J.J., Szalma, J., Hórvölgyi, Z. Langmuir 14 (1998) 6501. 